\title{
Teledentistry, DSM and the COVID-19 Pandemic: The Beginning of a New Era?
}

Jean-François Masse, DMD, MSc, FACD, Diplomate, ABDSM

Editor-in-Chief Journal of Dental Sleep Medicine Universite Laval, Quebec City, Quebec, Canada

In the last three months, the world has changed. On a personal note, I now wear a mask whenever I go out; my 83-year-old father, who barely used the Internet, now does his grocery shopping online and loves it; and my 92 year-old mother-in-law, who lives by herself in a nursing home, was not allowed to see any of us up until recently. As it seems that the number of new reported cases is not slowing down and new outbreaks of the coronavirus have appeared in China, chances are we will have to deal with this situation for longer than originally hoped.

On a professional note, we are facing a difficult challenge: how can we keep on delivering much-needed services while providing a safe environment for our patients, our staff and ourselves? The fear of getting the SARS-CoV2 may be as detrimental to our practice as the virus itself. Could teledentistry be part of the solution? All aspects of oral appliance therapy obviously cannot be done in front of a computer screen.

Nevertheless, there is room for a change in the way we practice. It is reasonable to think that many parts of oral appliance therapy are actually conducive to telehealth. We just have to have a look at what is happening on the medical side to realize the amplitude of the upcoming phenomenon...

In March 2020, the White House waived telemedicine requirements for Medicare patients. In the week ending March 7th, 11,000 members received telehealth services. In the week ending April 18th, almost 1.3 million members had received telehealth services - an increase of more than $11,718 \%$ in a month and a half.1 Forrester Research predicts that virtual care visits in America will soar to more than 1 billion this year.2 Even before the outbreak, the Cleveland Clinic predicted that by $2024,50 \%$ of medical visits will be virtual. 3 The change for telehealth is so sudden and rapid that one cannot look for recent relevant information in the traditional channels like Google Scholar and PubMed. We instead have to use reliable newsfeeds...

Even though telehealth evolves at the same insane in which speed new technologies arrive, we can make some conclusions about its use.
The advantages of virtually seeing patients, whenever possible, are numerous:

- Diminishes the risk of virus transmission.

- It is less costly for everyone: no need for PPEs, no need to disinfect the operatory, patient does not have to miss work or find childcare to come to the office.

- Allows out-of-town and rural patients to be seen more easily.

- Allows us to help fellow dentists in the field more easily.

On the other hand, the use of telemedicine raises some practical questions as well:

- What are ethical considerations when using virtual consultations? What are the limits where privacy is concerned? Should we record the entire consultation for liability?

- Is there a legal protocol proposed by a dental board, detailing all the steps necessary to follow through the virtual consultation?

- Will insurance companies cover telehealth visits?

- 42 million Americans do not have broadband internet.4 Should we be concerned about their lack of access to telehealth services?

I believe that time will help answer these questions. Nevertheless, the main take-away is that we will need to change some of our habits. This is never easy, but as more and more physicians and patients embrace this new paradigm and new technology like 5G, it opens possibilities for innovative thinking and cutting-edge treatment. Can we afford to not incorporate virtual consults in our protocols? Asking the question is answering it. Your comments are welcome. 


\section{CITATION}

Masse, JF. Teledentistry, DSM and the COVID-19 pandemic: The beginning of a new era?. J Dent Sleep Med. 2020;7(3)

\section{REFERENCES}

1. Pifer R. Medicare members using telehealth grew 120 times in early weeks of COVID-19 as regulations eased. Healthcare Dive. May 27. Accessed June 29, 2020. https://www.healthcaredive.com/news/medicare-seniorstelehealth-covid-coronavirus-cms-trump/578685/

2. US virtual care visits soar to more than 1 billion. Forrester. Forrester Research. April 10, 2020. Accessed June 29, 2020 https://go.forrester.com/press-newsroom/us-virtual-care-visits-tosoar-to-more-than-1-billion/

3. Cleveland Clinic, American Well Partner to Launch Digital Health Company. October 21, 2019. Retrieved June 29, 2020. https://newsroom.clevelandclinic.org/2019/10/21/clevelandclinic-american-well-partner-to-launch-digital-healthcompany/\#: : text=Cleveland\%20Clinic\%20projects\%20that $\% 20 \mathrm{i}$ n,Clinic\%20Express\%20Care\%C2\%AE\%20Online.

4. Poon L. There are far more Americans without broadband access than previously thought. Bloomberg. February 19, 2020. https://www.bloomberg.com/news/articles/2020-02-19/where-theu-s-underestimates-the-digital-divide

\section{SUBMISSION AND CORRESPONDENCE INFORMATION}

\section{Submitted in final revised form June 27, 2020.}

Address correspondence to: Jean-François Masse, DDS, MSc, FACD, D.ABDSM, Professor, Universite Laval, 2780 Masson \#200, Quebec City, QC, G1P 1J6, Canada; Tel: 418871-1447; Fax: 418-871-4983; Email: jean-francois.masse@fmd.ulaval.ca 